\title{
TITLE:
}

\section{Quantum-statistical mechanics of an atom-dimer mixture: Lee-Yang cluster expansion approach}

\section{$\operatorname{AUTHOR}(S)$ :}

Ohkuma, T; Ueda, M

\section{CITATION:}

Ohkuma, T ...[et al]. Quantum-statistical mechanics of an atom-dimer mixture: Lee-Yang cluster expansion approach. PHYSICAL REVIEW A 2006, 73(6): 063608.

\section{ISSUE DATE:}

2006-06

URL:

http://hdl.handle.net/2433/49965

\section{RIGHT:}

Copyright 2006 American Physical Society 
PHYSICAL REVIEW A 73, 063608 (2006)

\title{
Quantum-statistical mechanics of an atom-dimer mixture: Lee-Yang cluster expansion approach
}

\author{
Takahiro Ohkuma ${ }^{1}$ and Masahito Ueda ${ }^{2,3}$ \\ ${ }^{1}$ Yukawa Institute for Theoretical Physics, School of Science, Kyoto University, Kyoto 606-8502, Japan \\ ${ }^{2}$ Department of Physics, Tokyo Institute of Technology, 2-12-1 Ookayama, Meguro-ku, Tokyo 152-8551, Japan \\ ${ }^{3}$ ERATO, Japan Science and Technology Corporation (JST), Saitama 332-0012, Japan
}

(Received 2 February 2006; published 7 June 2006)

\begin{abstract}
We use the Lee-Yang cluster expansion method to study quantum-statistical properties of a mixture of interconvertible atoms and dimers, where the dimers form in a two-body bound state of the atoms. We point out an infinite series of cluster diagrams whose summation leads to the Bose-Einstein condensation of the dimers below a critical temperature. Our theory captures some important features of a cold atom-dimer mixture such as interconversion of atoms and dimers and properties of the mixture at the unitarity limit.
\end{abstract}

DOI: 10.1103/PhysRevA.73.063608

PACS number(s): 03.75.Hh, 05.30.-d

\section{INTRODUCTION}

One revolutionary aspect of ultracold alkali-metal gases is the tunability of interatomic interactions using a Feshbach resonance $[1,2]$. Weakly bound dimers have been created on the positive $a$ side of a Feshbach resonance for both bosons [3-5] and fermions [6-9], where $a$ is the $s$-wave scattering length. These dimers have bosonic characters and have experimentally been demonstrated to undergo Bose-Einstein condensation (BEC) below a critical temperature [10-12]. Because atoms and dimers are interconvertible due to quantum exchange effects and undergo BEC at different temperatures, the mixture of atoms and dimers offers an interesting new research arena in quantum-statistical many-body systems. However, first-principle approach to properties of the atom-dimer mixture, especially close to the Feshbach resonance, presents a formidable theoretical challenge because of the divergence of the scattering length.

In this paper we address this problem using the cluster expansion method of Lee and Yang [13]. This is a quantumstatistical cumulant expansion method that enables us to systematically expand the grand partition function in terms cluster integrals. In particular, we find an infinite serise of clusters whose summation leads to the BEC of dimers. We also investigate unitary behavior of the atom-dimer mixture at the limit of $a \rightarrow \infty$.

This paper is organized as follows. In Sec. II, we review the Lee-Yang cumulant expansion methods for quantum gases by restricting ourselves to one-particle and two-particle clusters. In Sec. III, we perform a partial summation of connected diagrams within an $s$-wave approximation to calculate grand partition functions of Bose and Fermi systems with arbitrary spin. In Sec. IV, we show how the BEC of strongly bounded dimers can be obtained with the present method. In Sec. V, we investigate the mixture of atoms and dimers in a high-temperature regime and discuss universal thermodynamics. In Sec. VI, we consider a possible coexsistence of the atomic and dimer BECs. In Sec. VII, we summarize the main results of this paper. Some complicated algebraic manipulations are relegated to the appendices to avoid disgressing from the main subjects.

\section{LEE-YANG CLUSTER EXPANSION}

We consider a system of $N$ atoms described by the Hamiltonian

$$
\mathcal{H}_{N}=\sum_{i=1}^{N} \frac{\mathbf{p}_{i}^{2}}{2 m}+\sum_{i<j} \mathcal{V}\left(\mathbf{r}_{i}-\mathbf{r}_{j}\right)
$$

where $\mathbf{p}_{i}$ is the momentum of the $i$ th particle and $\mathcal{V}$ is the two-body interaction which is assumed to be independent of spin variables and have spherical symmetry. According to the cumulant expansion method, the logarithm of the grand partition function is expanded as a power series of the fugacity $z=e^{\beta \mu}$, where $\mu$ is the chemical potential and $\beta=\left(k_{\mathrm{B}} T\right)^{-1}$ is the inverse of the Boltzmann constant $k_{\mathrm{B}}$ times the absolute temperature $T$. The $n$th order term in the cumulant expansion describes the $n$-body problem of the system.

In a quantum Boltzmann gas, the coefficient of the $n$th order term is contributed only from connected clusters of $n$ particles $[14,15]$. The first-order term is contributed from the one-body cluster

$$
U_{1}=e^{-\beta \mathcal{H}_{1}}
$$

which describes the noninteracting part of the system, where $\mathcal{H}_{1}=\mathbf{p}^{2} / 2 m$ is the one-body part of the Hamiltonian. The higher-order clusters $U_{2}, U_{3}, \ldots$, are defined in terms of the Boltzmann factor for the Hamiltonian $\mathcal{H}_{n}$

$$
W_{n}=e^{-\beta \mathcal{H}_{n}}
$$

as

$$
\begin{aligned}
\left\langle 1^{\prime}, 2^{\prime}\left|W_{2}\right| 1,2\right\rangle=\left\langle 1^{\prime}\left|U_{1}\right| 1\right\rangle\left\langle 2^{\prime}\left|U_{1}\right| 2\right\rangle+\left\langle 1^{\prime}, 2^{\prime}\left|U_{2}\right| 1,2\right\rangle & \\
\left\langle 1^{\prime}, 2^{\prime}, 3^{\prime}\left|W_{3}\right| 1,2,3\right\rangle= & \left\langle 1^{\prime}\left|U_{1}\right| 1\right\rangle\left\langle 2^{\prime}\left|U_{1}\right| 2\right\rangle\left\langle 3^{\prime}\left|U_{1}\right| 3\right\rangle \\
& +\left\langle 1^{\prime}\left|U_{1}\right| 1\right\rangle\left\langle 2^{\prime}, 3^{\prime}\left|U_{2}\right| 2,3\right\rangle+\left\langle 2^{\prime}\left|U_{1}\right| 2\right\rangle \\
& \times\left\langle 1^{\prime}, 3^{\prime}\left|U_{2}\right| 1,3\right\rangle+\left\langle 3^{\prime}\left|U_{1}\right| 3\right\rangle \\
& \times\left\langle 1^{\prime}, 2^{\prime}\left|U_{2}\right| 1,2\right\rangle \\
& +\left\langle 1^{\prime}, 2^{\prime}, 3^{\prime}\left|U_{3}\right| 1,2,3\right\rangle \\
& \quad \text { etc. }
\end{aligned}
$$

where 1 and $1^{\prime}$ stand for one-particle states of the first particle, 2 and $2^{\prime}$ stand for those of the second particle, etc. Thus the $n$-body cluster, $\left\langle 1^{\prime}, 2^{\prime}, \ldots, n^{\prime}\left|U_{n}\right| 1,2, \ldots, n\right\rangle$, represents the remainder of the Boltzmann factor of the $n$-body Hamiltonian, $\left\langle 1^{\prime}, 2^{\prime}, \ldots, n^{\prime}\left|e^{-\beta \mathcal{H}_{n}}\right| 1,2, \ldots, n\right\rangle$, from which 
all separable smaller clusters are removed. We call this remainder the $n$-particle connected cluster or simply the $n$-particle cluster. In terms of $U_{n}$, the grand partition function $\Xi$ is given by (see Appendix A for derivation)

$$
\ln \Xi=\sum_{n=1}^{\infty} \frac{z^{n}}{n !} \operatorname{Tr}\left(U_{n}\right)
$$

where $z=e^{\beta \mu}$ is the fugacity and $\operatorname{Tr}\left(U_{n}\right)$ denotes the trace of $U_{n}$.

In quantum statistical mechanics, in contrast, the $n$th order coefficient involves not only $n$-particle connected clusters but also smaller clusters that are entangled due to symmetrization or antisymmetrization of identical bosons or fermions [13]. For bosons, symmetrization is carried out through summation over all permutations of the particle configuration $1^{\prime}, 2^{\prime}, \ldots, n^{\prime}$. Then we get the restricted Boltzmann factor as

$$
\left\langle 1^{\prime}, \ldots, n^{\prime}\left|W_{n}^{\mathrm{S}}\right| 1, \ldots, n\right\rangle=\sum_{\mathfrak{P}}\left\langle\mathfrak{P}\left(1^{\prime}\right), \ldots, \mathfrak{P}\left(n^{\prime}\right)\left|W_{n}\right| 1, \ldots, n\right\rangle,
$$

where $\mathfrak{P}\left(1^{\prime}\right), \ldots, \mathfrak{P}\left(n^{\prime}\right)$ is a permutation of $1^{\prime}, \ldots, n^{\prime}$ and the sum runs over all possible permutations. For fermions, antisymmetrization is carried out as

$$
\begin{aligned}
\left\langle 1^{\prime}, \ldots, n^{\prime}\left|W_{n}^{\mathrm{A}}\right| 1, \ldots, n\right\rangle= & \sum_{\mathfrak{P}} \operatorname{sgn} \mathfrak{P}\left\langle\mathfrak{P}\left(1^{\prime}\right), \ldots, \mathfrak{P}\left(n^{\prime}\right)\right. \\
& \left.\times\left|W_{n}\right| 1, \ldots, n\right\rangle,
\end{aligned}
$$

where $\operatorname{sgn} \mathfrak{P}=1$ if $\mathfrak{P}$ is an even permutation and $\operatorname{sgn} \mathfrak{P}$ $=-1$ if $\mathfrak{P}$ is an odd permutation. In terms of the restricted Boltzmann factors $W_{n}^{\mathrm{S}, \mathrm{A}}$, we define Lee-Yang clusters $U_{n}^{\mathrm{S}, \mathrm{A}}$ as

$$
\begin{aligned}
&\left\langle 1^{\prime}\left|W_{1}^{\mathrm{S}, \mathrm{A}}\right| 1\right\rangle=\left\langle 1^{\prime}\left|U_{1}^{\mathrm{S}, \mathrm{A}}\right| 1\right\rangle, \\
&\left\langle 1^{\prime}, 2^{\prime}\left|W_{2}^{\mathrm{S}, \mathrm{A}}\right| 1,2\right\rangle=\left\langle 1^{\prime}\left|U_{1}^{\mathrm{S}, \mathrm{A}}\right| 1\right\rangle\left\langle 2^{\prime}\left|U_{1}^{\mathrm{S}, \mathrm{A}}\right| 2\right\rangle+\left\langle 1^{\prime}, 2^{\prime}\left|U_{2}^{\mathrm{S}, \mathrm{A}}\right| 1,2\right\rangle, \\
&\left\langle 1^{\prime}, 2^{\prime}, 3^{\prime}\left|W_{3}^{\mathrm{S}, \mathrm{A}}\right| 1,2,3\right\rangle=\left\langle 1^{\prime}\left|U_{1}^{\mathrm{S}, \mathrm{A}}\right| 1\right\rangle\left\langle 2^{\prime}\left|U_{1}^{\mathrm{S}, \mathrm{A}}\right| 2\right\rangle\left\langle 3^{\prime}\left|U_{1}^{\mathrm{S}, \mathrm{A}}\right| 3\right\rangle \\
&+\left\langle 1^{\prime}\left|U_{1}^{\mathrm{S}, \mathrm{A}}\right| 1\right\rangle\left\langle 2^{\prime}, 3^{\prime}\left|U_{2}^{\mathrm{S}, \mathrm{A}}\right| 2,3\right\rangle \\
&+\left\langle 2^{\prime}\left|U_{1}^{\mathrm{S}, \mathrm{A}}\right| 2\right\rangle\left\langle 1^{\prime}, 3^{\prime}\left|U_{2}^{\mathrm{S}, \mathrm{A}}\right| 1,3\right\rangle \\
&+\left\langle 3^{\prime}\left|U_{1}^{\mathrm{S}, \mathrm{A}}\right| 3\right\rangle\left\langle 1^{\prime}, 2^{\prime}\left|U_{2}^{\mathrm{S}, \mathrm{A}}\right| 1,2\right\rangle \\
&+\left\langle 1^{\prime}, 2^{\prime}, 3^{\prime}\left|U_{3}^{\mathrm{S}, \mathrm{A}}\right| 1,2,3\right\rangle,
\end{aligned}
$$

etc.

Because of (anti)symmetrization, Lee-Yang clusters $U_{n}^{\mathrm{S}, \mathrm{A}}$ with $n \geq 2$, in general, do not vanish even for ideal bosons (fermions). For the case of $n=2$, for example, we have $\left\langle 1^{\prime}, 2^{\prime}\left|U_{2}^{\mathrm{S}, \mathrm{A}}\right| 1,2\right\rangle= \pm\left\langle 2^{\prime}\left|U_{1}^{\mathrm{S}, \mathrm{A}}\right| 1\right\rangle\left\langle 1^{\prime}\left|U_{1}^{\mathrm{S}, \mathrm{A}}\right| 2\right\rangle$, where the plus (minus) sign applies to bosons (fermions).

In terms of $U_{n}^{\mathrm{S}, \mathrm{A}}$, the grand partition function $\Xi$ is written as (a)

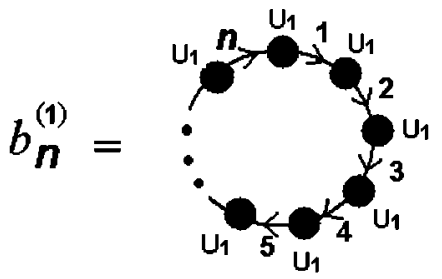

(b)

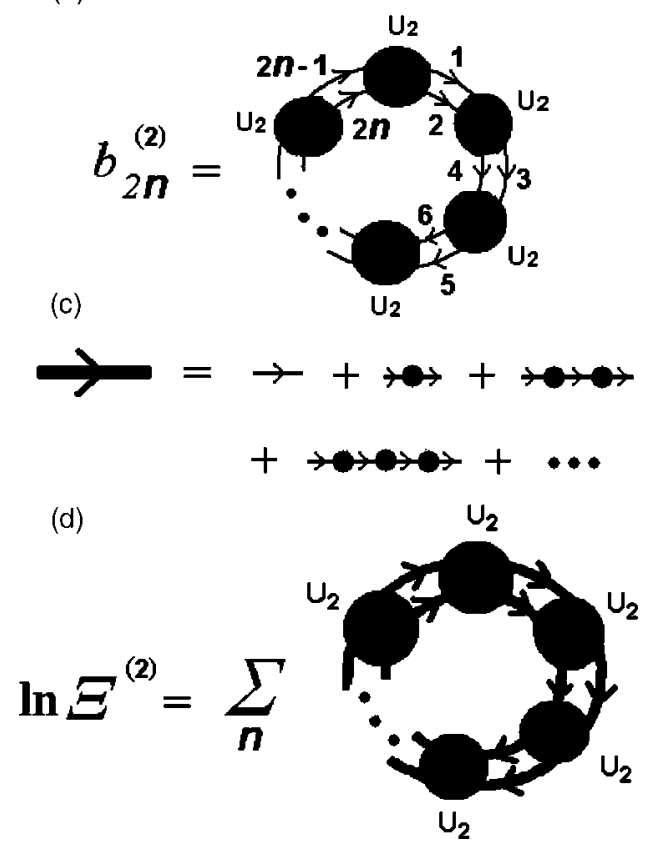

FIG. 1. Schematic diagrams of cluster expansions. (a) Oneparticle connected diagram, where each dot represents $U_{1}$ which describes the quantum exchange effect. (b) An example of the connected diagrams of two-particle clusters, where each dot represents $U_{2}$ which describes the exact two-body scattering but not the quantum exchange effect. We distinguish $U_{1}$ and $U_{2}$ by the number of the incoming and outgoing lines. (c) A thick line represents the sum of all one-particle connected diagrams. A mathematical expression of this replacement is given in Eq. (27). (d) Corrections to the two-body clusters due to the quantum exchange effect. Such corrections can be taken into account through the replacement of thin lines by thick ones.

$$
\ln \Xi=V \sum_{n=1}^{\infty} b_{n} z^{n}
$$

where $V$ is the volume of the system and

$$
b_{n}=\frac{1}{V n !} \operatorname{Tr}\left(U_{n}^{\mathrm{S}, \mathrm{A}}\right)
$$

is the Lee-Yang cluster integral. Once $\ln \Xi$ is obtained, the equation of state and the average number density are given by

$$
\beta p=\lim _{V \rightarrow \infty} \frac{\ln \Xi}{V}
$$

and 


$$
\rho=\lim _{V \rightarrow \infty} \frac{1}{V} \frac{\partial \ln \Xi}{\partial \ln z},
$$

respectively, where $p$ is the pressure and $\rho$ is the number density of the system. Examples of the Lee-Yang diagrams are schematically illustrated in Fig. 1.

For noninteracting bosons or fermions, the grand partition function $\Xi^{(1)}$ is written as

$$
\frac{1}{V} \ln \Xi^{(1)}=\sum_{n=1}^{\infty} b_{n}^{(1)} z^{n}
$$

where $b_{n}^{(1)}$ is the cluster integral which is represented in terms of wave vector $\mathbf{k}$ and spin $\sigma$ as

$$
b_{n}^{(1)}=\frac{P^{n-1}(n-1) !}{V n !} \sum_{\mathbf{k}, \sigma}\left\langle\mathbf{k}, \sigma\left|U_{1}^{n}\right| \mathbf{k}, \sigma\right\rangle .
$$

In Eq. (17), $U_{1}$ represents the one-particle cluster and the factor $(n-1)$ ! is the number of topologically distinct configurations of $n$ particles that give the same contribution. The sign function $P$ takes on +1 for bosons and -1 for fermions.

Each dot in Fig. 1(a) represents the one-particle cluster $U_{1}$ which is connected with one incoming line corresponding to a ket vector and one outgoing line corresponding to a bra- vector. To be explicit, we rewrite the coefficient $b_{n}^{(1)}$ in Eq. (17) by inserting the resolution of unity $1=\Sigma_{\mathbf{k}, \sigma}|\mathbf{k}, \sigma\rangle\langle\mathbf{k}, \sigma|$ between the $U_{1}$ operators, with the following result:

$$
\begin{aligned}
b_{n}^{(1)}= & \frac{P^{n-1}(n-1) !}{V n !} \sum_{\left\{\mathbf{k}_{i} \sigma_{i}\right\}}\left\langle\mathbf{k}_{1}, \sigma_{1}\left|U_{1}\right| \mathbf{k}_{n}, \sigma_{n}\right\rangle \cdots\left\langle\mathbf{k}_{3}, \sigma_{3}\left|U_{1}\right| \mathbf{k}_{2}, \sigma_{2}\right\rangle \\
& \times\left\langle\mathbf{k}_{2}, \sigma_{2}\left|U_{1}\right| \mathbf{k}_{1}, \sigma_{1}\right\rangle
\end{aligned}
$$

where $\left\langle\mathbf{k}_{j}, \sigma_{j}\left|U_{1}\right| \mathbf{k}_{i}, \sigma_{i}\right\rangle$ is the transition amplitude from the $i$ th particle state to the $j$ th one and thus describes the effect of the quantum exchange between identical particles. It is the ring diagrams of $U_{1}$ as illustrated in Fig. 1(a) that bring the system to quantum degeneracy of atoms (but not dimers). Substituting Eq. (17) into Eq. (16), we obtain

$$
\ln \Xi^{(1)}=-P \operatorname{Tr} \ln \left(1-P z U_{1}\right) .
$$

When a two-body interaction exists, two-particle clusters and larger ones appear in the cumulant expansion in addition to one-particle clusters. There are $n$ incoming lines and $n$ outgoing lines associated with an $n$-particle cluster. Formally, we can write down the coefficient $b_{n}$ as a sum over all possible connected diagrams:

$$
b_{n}=\frac{1}{V n !} \operatorname{Tr} \sum_{\left\{m_{j}\right\}} \sum_{\mathfrak{P}^{\prime}}\left(\begin{array}{c}
1 \\
\operatorname{sgn} \mathfrak{P}^{\prime}
\end{array}\right) \underbrace{\left\langle\mathfrak{a}^{\prime}\left|U_{1}\right| a\right\rangle \cdots\left\langle\mathfrak{b}^{\prime}\left|U_{1}\right| b\right\rangle}_{m_{1}} \underbrace{\left\langle\mathfrak{c}^{\prime}, \mathfrak{d}^{\prime}\left|U_{2}\right| c, d\right\rangle \cdots\left\langle\mathfrak{e}^{\prime}, \mathfrak{f}^{\prime}\left|U_{2}\right| e, f\right\rangle}_{m_{2}} \cdots \underbrace{\left\langle\mathfrak{g}^{\prime}, \mathfrak{h}^{\prime}, \ldots, \mathfrak{i}^{\prime}\left|U_{n}\right| g, h, \ldots, i\right\rangle}_{m_{n}},
$$

where the set $\left\{m_{j}\right\}$ satisfies $\sum_{j=1}^{n} j m_{j}=n$, the sum over $\mathfrak{P}^{\prime}$ is to be taken over all possible permutations $\mathfrak{P}^{\prime}$ of particle configurations, $\mathfrak{a}^{\prime}, \mathfrak{b}^{\prime}, \ldots$, for each set $\left\{m_{j}\right\}$, and $\mathfrak{a}^{\prime}, a, \mathfrak{b}^{\prime}, b$, etc., symbolically represent a certain set of wavevector and spin states. In the round brackets of Eq. (20), 1 and sgn $\mathfrak{P}^{\prime}$ correspond to bosons and fermions, respectively.

In a uniform system, $U_{n}$ in the wave vector representation involves Kronecker's $\delta$ function arising from the conservation of the total momentum. In the thermodynamic limit $V$ $\rightarrow \infty$, we have

$$
\begin{aligned}
& \left\langle\mathbf{k}_{1}^{\prime} \sigma_{1}^{\prime}, \ldots, \mathbf{k}_{n}^{\prime} \sigma_{n}^{\prime}\left|U_{n}\right| \mathbf{k}_{1} \sigma_{1}, \ldots, \mathbf{k}_{n} \sigma_{n}\right\rangle \\
& \quad=\delta^{3}\left(\mathbf{K}^{\prime}-\mathbf{K}\right)\left(\prod_{j=1}^{n} \delta_{\sigma_{j}^{\prime}, \sigma_{j}}\right) u_{n}\left(\mathbf{k}_{1}^{\prime}, \ldots, \mathbf{k}_{n}^{\prime}, \mathbf{k}_{1}, \ldots, \mathbf{k}_{n}\right)
\end{aligned}
$$

where $\mathbf{K}^{\prime}=\Sigma_{i} \mathbf{k}_{i}^{\prime}$ and $\mathbf{K}=\Sigma_{i} \mathbf{k}_{i}$, and $u_{n}$ is independent of the volume. Furthermore, the trace operation, Tr, is to be interpreted as an integral over the wave vector, $\int d \mathbf{k}$, and the $\delta$ function should be interpreted as $\lim _{V \rightarrow \infty} \frac{V}{(2 \pi)^{3}} \delta_{\mathbf{K}^{\prime}, \mathbf{K}}$. Thus, in the thermodynamic limit,

$$
\begin{aligned}
\frac{1}{V} \operatorname{Tr}\left(U_{n}\right) & =\frac{1}{V_{\mathbf{k}_{1}, \ldots, \mathbf{k}_{n}}}\left\langle\mathbf{k}_{1}, \ldots, \mathbf{k}_{n}\left|U_{n}\right| \mathbf{k}_{1}, \ldots, \mathbf{k}_{n}\right\rangle \\
& \rightarrow \frac{1}{(2 \pi)^{3}}\left(\prod_{j=1}^{n} \int d \mathbf{k}_{j}\right) u_{n}\left(\mathbf{k}_{1}, \ldots, \mathbf{k}_{n}\right)
\end{aligned}
$$

The same argument applies to quantum clusters $U_{n}^{\mathrm{S}, \mathrm{A}}$.

\section{REPEATED BINARY COLLISIONS}

We first discuss the case of spinless bosons, and then extend it to cases of spin- $J$ bosons and fermions. Throughout this paper we take into account many-body effects up to two-particle clusters and make the $s$-wave approximation. This assumption is valid up to the order of $o\left((k a)^{3}\right)$, provided that $k a<1$, where $a$ is the $s$-wave scattering length and $k$ is a characteristic wave number of the system. Within the $s$-wave approximation the wave number representation of a quantum state of a uniform system can be decomposed into the part that involves only the absolute value of the relative momentum $\left|\mathbf{k}-\mathbf{k}^{\prime}\right|$ and the part that involves only the centerof-mass momentum as 


$$
\left\langle\mathbf{k}, \mathbf{k}^{\prime} \mid \psi\right\rangle=\Psi\left(\mathbf{k}+\mathbf{k}^{\prime}\right) \psi\left(\left|\mathbf{k}-\mathbf{k}^{\prime}\right|\right) .
$$

Thus, the exchange between $\mathbf{k}$ and $\mathbf{k}^{\prime}$ yields an identical result, and so we need not distingush between $\left|\mathbf{k}, \mathbf{k}^{\prime}\right\rangle$ and $\left|\mathbf{k}^{\prime}, \mathbf{k}\right\rangle$.

We consider a set of connected diagrams that describe repeated collisions between two particles, as illustrated in Fig. 1(b). Each dot stands for the $U_{2}$ cluster and hence represents the exact two-body scattering unlike the case of the ladder approximation. Thus if the two-body problem can be solved exactly, the present method offers a powerful tool for computing the partition function accurately. When the twobody problem supports a bound state, the dot should also describe dimers in addition to two-body scattering.

The $U_{2}$ cluster is expressed in operator form as

$$
U_{2}=e^{-\beta \mathcal{H}_{2}}-e^{-\beta \mathcal{H}_{1}} \otimes e^{-\beta \mathcal{H}_{1}}
$$

where $\mathcal{H}_{1}$ and $\mathcal{H}_{2}$ are the one-body Hamiltonian and the two-body Hamiltonian, respectively. This definition agrees with that of the $U_{2}$ cluster in Eq. (4). Let $K_{2 n}$ be the number of diagrams that give the same contribution in the $2 n$-body cumulant consisting of $U_{2}$ 's only. To construct the corresponding diagram shown in Fig. 1(b), we must choose $n$ pairs of lines, each constituting a two-particle cluster. The number of ways to choose two lines for the first pair is ${ }_{2 n} C_{2}$, it is ${ }_{2 n-2} C_{2}$ for the second pair, and so on. Since a factor of 2 arises from each pair due to the exchange between $\mathbf{k}$ and $\mathbf{k}^{\prime}$ [see the remark below Eq. (23)], we have, in total, an additional factor of $2^{n}$. Thus

$$
K_{2 n}={ }_{2 n} C_{2} \times{ }_{2 n-2} C_{2} \times \cdots \times{ }_{2} C_{2} 2^{n} n^{-1}=\frac{(2 n) !}{n},
$$

where the factor $n^{-1}$ accounts for the fact that the cyclic permutation of $n$ pairs yields $n$ topologically identical diagrams which should be counted just once. The contribution to the grand partition function from binary collisions is thus calculated to be

$$
\begin{aligned}
& \sum_{n=1}^{\infty} \frac{z^{2 n}}{(2 n) !} K_{2 n} \int d \mathbf{k}_{1} d \mathbf{k}_{2}\left\langle\mathbf{k}_{1}, \mathbf{k}_{2}\left|U_{2}^{n}\right| \mathbf{k}_{1}, \mathbf{k}_{2}\right\rangle \\
& \quad=-\int d \mathbf{k}_{1} d \mathbf{k}_{2}\left\langle\mathbf{k}_{1}, \mathbf{k}_{2}\left|\ln \left[1-z^{2} U_{2}\right]\right| \mathbf{k}_{1}, \mathbf{k}_{2}\right\rangle \\
& \quad=-\operatorname{Tr} \ln \left[1-z^{2} U_{2}\right] .
\end{aligned}
$$

Equation (26) is derived under the assumption that two outgoing lines go directly from one $U_{2}$ into another without undergoing quantum exchange with other atoms. However, Nature does not distinguish between colliding atoms and their surrounding atoms, and therefore one-particle lines connecting $U_{2}$ 's should be renormalized to take into account the quantum exchange effect described by $U_{1}$. The number of times that $U_{1}$ acts on a single line ranges from zero to infinity. Thus the single-particle bare propagator should be renormalized as

$$
\begin{array}{cc}
z \int d i|i\rangle\langle i| & \text { (bare) } \\
\rightarrow \sum_{n=0}^{\infty} z^{n+1} \int d i \int d j|j\rangle\left\langle j\left|U_{1}^{n}\right| i\right\rangle\langle i| & \text { (renormalized). }
\end{array}
$$

In Fig. 1(c), we schematically illustrate the bare propagator as a thin line and the renormalized single-particle propagator as a thick line.

In accordance with this renormalization procedure, we replace thin one-particle lines in Fig. 1(b) by thick ones and obtain the corresponding grand partition function as schematically illustrated in Fig. 1(d). To implement this procedure algebraically, suppose that there are $m$ "surrounding" atoms in addition to the $2 n$ particles constituting the twoparticle cluster ring. In this case, $K_{2 n}$ in Eq. (25) should be replaced with

$$
K_{2 n+m}={ }_{2 n+m} C_{2} \times \cdots \times{ }_{2+m} C_{2} 2^{n} n^{-1} m !=\frac{(2 n+m) !}{n},
$$

where $m$ ! accounts for the number of orderings of the surrounding atoms. The second-order correction, $\ln \Xi^{(2)}$, to the grand partition function is thus given as

$$
\begin{aligned}
\ln \Xi^{(2)}= & \sum_{n=1}^{\infty} \sum_{m=0}^{\infty} \sum_{m=m_{1}+\cdots+m_{2 n}} \frac{z^{2 n+m}}{(2 n+m) !} K_{2 n+m} \\
& \times \operatorname{Tr}\left\{\left(U_{1}^{m_{1}} \otimes U_{1}^{m_{2}} U_{2}\right) \times \cdots \times\left(U_{1}^{m_{2 n-1}} \otimes U_{1}^{m_{2 n}} U_{2}\right)\right\} \\
= & -\operatorname{Tr} \ln \left[1-f_{\mathrm{B}}\left(\mathcal{H}_{1}\right) \otimes f_{\mathrm{B}}\left(\mathcal{H}_{1}\right)\left(U_{1}^{-1} \otimes U_{1}^{-1} U_{2}\right)\right], \quad(29)
\end{aligned}
$$

where $f_{\mathrm{B}}\left(\mathcal{H}_{1}\right)=\left[\exp \left\{\beta\left(\mathcal{H}_{1}-\mu\right)\right\}-1\right]^{-1}$ is the Bose-Einstein distribution operator. Introducing the $S$ matrix, $\mathcal{S}$, as

$$
\mathcal{S}=1+U_{1}^{-1} \otimes U_{1}^{-1} U_{2},
$$

we have

$$
\ln \Xi^{(2)}=-\operatorname{Tr} \ln \left[1-\left(f_{\mathrm{B}} \otimes f_{\mathrm{B}}\right)(\mathcal{S}-1)\right] .
$$

Substituting Eq. (24) into Eq. (30), we obtain

$\mathcal{S}=\left(e^{\beta \mathcal{H}_{1}} \otimes e^{\beta \mathcal{H}_{1}}\right) e^{-\beta \mathcal{H}_{2}}=T_{\tau} \exp \left(-\int_{0}^{\beta} \mathcal{V}(\tau) d \tau\right)$,

where $T_{\tau}$ is the imaginary-time ordering operator and

$$
\mathcal{V}(\tau) \equiv\left(e^{\tau \mathcal{H}_{1}} \otimes e^{\tau \mathcal{H}_{1}}\right) \mathcal{V}\left(e^{-\tau \mathcal{H}_{1}} \otimes e^{-\tau \mathcal{H}_{1}}\right)
$$

with $\mathcal{V}=\mathcal{H}_{2}-\mathcal{H}_{1} \otimes 1-1 \otimes \mathcal{H}_{1}$. Comparing Eq. (31) with Eq. (26), we find that the quantum exchange effect amounts to the replacement of the fugacity $z$ with the distribution function $f_{\mathrm{B}}$ and $U_{2}$ with $\mathcal{S}-1$. It follows from Eqs. (19) and (31) that the grand partition function for spinless bosons is given by

$$
\begin{aligned}
\ln \Xi_{\mathrm{B}}^{J=0}= & \ln \Xi^{(1)}+\ln \Xi^{(2)} \\
= & -\operatorname{Tr} \ln \left[1-z U_{1}\right]-\operatorname{Tr} \ln \left\{1-\left[f_{\mathrm{B}}\left(\mathcal{H}_{1}\right) \otimes f_{\mathrm{B}}\left(\mathcal{H}_{1}\right)\right]\right. \\
& \times(\mathcal{S}-1)\} .
\end{aligned}
$$


As shown in Appendix B, this result can be generalized to the case of spin- $J$ bosons and fermions:

$$
\begin{aligned}
\ln \Xi_{\mathrm{B}}= & -(2 J+1) \operatorname{Tr} \ln \left[1-z U_{1}\right]-(2 J+1)(J+1) \\
& \times \operatorname{Tr} \ln \left[1-\left(f_{\mathrm{B}} \otimes f_{\mathrm{B}}\right)(\mathcal{S}-1)\right], \\
\ln \Xi_{\mathrm{F}}= & (2 J+1) \operatorname{Tr} \ln \left[1+z U_{1}\right]-J(2 J+1) \operatorname{Tr} \ln \left[1-\left(f_{\mathrm{F}} \otimes f_{\mathrm{F}}\right)\right. \\
& \times(\mathcal{S}-1)] .
\end{aligned}
$$

In Eqs. (35) and (36), Tr implies the trace operation only over the coordinate or the wavevector.

\section{BEC OF DIMERS}

We can use Eqs. (34)-(36) to investigate the BEC transition of dimers formed in the bound state of the two-body Hamiltonian for both Bose and Fermi systems. We first consider a system of spinless bosons, and assume that the twobody interaction $\mathcal{V}\left(\mathbf{r}_{i j}\right)$ is finite ranged and supports one bound state that describes a dimer state. The corresponding quantities in the Fermi system can be obtained by the replacement of the distribution function $f_{\mathrm{B}}$ with $f_{\mathrm{F}}$ or, equivalently, the fugacity $z$ with $-z$. We will consider the case of nonzero spin later.

When the $s$-wave scattering length $a$ is much larger than the effective range $l_{\text {eff }}$ of interaction, the binding energy of the dimer is given by $[16,17]$ [see also Eq. (45) below]

$$
E_{\mathrm{b}}=-\frac{\hbar^{2}}{m a^{2}}
$$

where $m$ is the mass of the atom, and $a$ is the $s$-wave scattering length. In this section, we assume that the binding energy is much larger than the thermal energy, i.e., $\left|E_{\mathrm{b}}\right| \gg k_{\mathrm{B}} T$. Thus we consider a parameter regime $l_{\text {eff }} \ll a \ll \lambda_{\mathrm{dB}}$, where $\lambda_{\mathrm{dB}} \equiv h / \sqrt{2 \pi m k_{\mathrm{B}} T}$ is the thermal de Broglie length.

We first note that the operator in the trace of Eq. (31) can be expanded as

$$
\left(f_{\mathrm{B}} \otimes f_{\mathrm{B}}\right)(\mathcal{S}-1)=\sum_{n_{1}=0}^{\infty} \sum_{n_{2}=0}^{\infty} z^{n_{1}+n_{2}+2} e^{-n_{1} \beta \mathcal{H}_{1}} e^{-n_{2} \beta \mathcal{H}_{1}} U_{2} .
$$

When the dimers are deeply bound so that $e^{\beta\left|E_{\mathrm{b}}\right|} \gg 1$, the two-particle cluster $U_{2}$ can be approximated as

$$
\begin{aligned}
U_{2}= & e^{-\beta \mathcal{H}_{\mathrm{G}}}\left(e^{-\beta\left(\mathbf{p}_{\mathrm{r}}^{2} / m+\mathcal{V}\right)}-e^{-\beta\left(\mathbf{p}_{\mathrm{r}}^{2} / m\right)}\right) \\
= & e^{-\beta \mathcal{H}_{\mathrm{G}}}\left(|\mathrm{b}\rangle e^{\beta\left|E_{\mathrm{b}}\right|}\left\langle\mathrm{b}\left|+\sum_{\mathbf{k}_{s}}\right| \mathbf{k}_{s}\right\rangle e^{-\beta \hbar^{2} \mathbf{k}_{s}^{2} / m}\left\langle\mathbf{k}_{s}\right|\right. \\
& \left.-\sum_{\mathbf{k}}|\mathbf{k}\rangle e^{-\beta \hbar^{2} \mathbf{k}^{2} / m}\langle\mathbf{k}|\right) \cong e^{-\beta \mathcal{H}_{\mathrm{G}}}|\mathrm{b}\rangle e^{\beta\left|E_{\mathrm{b}}\right|}\langle\mathrm{b}|,
\end{aligned}
$$

where $|\mathrm{b}\rangle$ represents the bound state, $\mathcal{H}_{\mathrm{G}}$ describes the center-of-mass part of the two-body Hamiltonian $\mathcal{H}_{2}$, and $\mathbf{p}_{\mathrm{r}}$ represents the momentum of the relative motion. The chemical potential must take such values that the geometrical series in Eq. (26) or Eq. (29) converges, that is,

$$
2 \mu \leq \inf \left\{\text { eigenvalue of } \mathcal{H}_{2}\right\} .
$$

Then the fugacity becomes very small, $z=e^{\beta \mu}$ $\leq \exp \left(-\beta\left|E_{\mathrm{b}}\right| / 2\right) \ll 1$, since by assumption $\beta\left|E_{\mathrm{b}}\right| \gg 1$. This allows us to ignore the quantum exchange effect for atoms (but not for dimers) and take only the term with $n_{1}=n_{2}=0$ in Eq. (38). Since the center-of-mass motion and the relative motion are decoupled from to each other, Eq. (31) reduces to

$$
\ln \Xi^{(2)}=-\operatorname{Tr} \ln \left[1-z^{2} e^{-\beta \mathcal{H}_{\mathrm{G}}}|b\rangle e^{\beta\left|E_{\mathrm{b}}\right|}\langle b|\right] .
$$

The contribution from free (unbound) atoms is also neglected due to the smallness of the fugacity. Then the average number of dimers is calculated to be

$$
\begin{aligned}
N^{\text {dimer }} & =\frac{1}{2} z \frac{\partial}{\partial z} \ln \Xi=\operatorname{Tr} \frac{1}{e^{\beta\left(\mathcal{H}_{\mathrm{G}}+E_{\mathrm{b}}-2 \mu\right)}-1} \\
& =N_{0}^{\mathrm{dimer}}+\frac{V}{(2 \pi)^{3}} \int d \mathbf{K} \frac{1}{e^{\beta\left(\hbar^{2} \mathbf{K}^{2} / 4 m+E_{\mathrm{b}}-2 \mu\right)}-1},
\end{aligned}
$$

where $N_{0}^{\text {dimer }}$ denotes the number of dimers in the $\mathbf{K}=\mathbf{0}$ state which is dropped when we replace the discrete sum with the integral.

The integral in the last term of Eq. (42) takes the maximum when the chemical potential is maximal. If the integral is below the total number of particles of the system, the remaining dimers should condense in the lowest energy (i.e., $\mathbf{K}=\mathbf{0})$ state. The transition temperature is given by the temperature at which the number of exited particles with $2 \mu$ $=E_{\mathrm{b}}$ is equal to the total number of dimers

$$
\begin{aligned}
\frac{N^{\text {dimer }}}{V} & =\int_{0}^{\infty} \frac{4 \pi K^{2} d K}{(2 \pi)^{3}} \frac{1}{e^{\beta_{\mathrm{c}} \hbar^{2} K^{2} / 4 m}-1} \\
& =\frac{2 \pi}{(2 \pi)^{3}}\left[\frac{4 m}{\beta_{\mathrm{c}} \hbar^{2}}\right]^{3 / 2} \Gamma\left(\frac{3}{2}\right) \zeta\left(\frac{3}{2}\right) .
\end{aligned}
$$

Hence the critical temperature is given by

$$
T_{\mathrm{c}}^{\text {dimer }}=\frac{2 \pi \hbar^{2}}{(2 m) k_{\mathrm{B}}}\left[\frac{N^{\text {dimer }}}{\zeta(3 / 2) V}\right]^{2 / 3} .
$$

This formula is identical to that of free dimers. Thermodynamic properties of dimers are also similar to those of free bosons. While we have assumed here that dimers are deeply bound, this assumption is by no means essential for the analysis of our grand partition function. At least in principle we can investigate the case in which the scattering length is comparable with or much larger than the thermal de Broglie length.

\section{HIGH-TEMPERATURE EXPANSION AND UNIVERSAL THERMODYNAMICS}

In a high-temperature regime, excited atoms and dimers can coexist. On the other hand, the two-body scattering probability decreases with decreasing $\rho \lambda_{\mathrm{dB}}^{3}$. By expanding the grand partition function (31) with respect to the fugacity, we can evaluate the quantum-statistical weight of dimers in the high-temperature regime.

To investigate this problem, we use a pseudopotential [18] 


$$
\mathcal{V}_{\text {pseudo }}(\mathbf{r})=\frac{4 \pi \hbar^{2}}{m} a \delta^{3}(\mathbf{r}) \frac{\partial}{\partial r} r .
$$

The advantage of using this pseudopotential is that we can exactly solve the two-body problem within the $s$-wave approximation. The pseudopotential (45) supports continuous scattering states and one bound state with the binding energy given in Eq. (37). With this pseudopotential, the two-particle cluster $U_{2}$ and the renormalized cluster $f_{\mathrm{B}} f_{\mathrm{B}}(\mathcal{S}-1)$, which includes an infinite series of fugacity, can be computed as done in Appendix C. Expanding the grand partition function up to the second order in the fugacity, we obtain the equation of state

$$
\begin{aligned}
& \beta p=\frac{1}{\lambda_{\mathrm{dB}}^{3}}\left[z+\frac{z^{2}}{2^{5 / 2}}+2 \sqrt{2}\left(e^{x^{2}}-\frac{1}{2} e^{x^{2}} \operatorname{erfc}(x)\right) z^{2}\right], \\
& \rho=\frac{1}{\lambda_{\mathrm{dB}}^{3}}\left[z+\frac{z^{2}}{2^{3 / 2}}+4 \sqrt{2}\left(e^{x^{2}}-\frac{1}{2} e^{x^{2}} \operatorname{erfc}(x)\right) z^{2}\right],
\end{aligned}
$$

where $x=\lambda_{\mathrm{dB}} /(\sqrt{2 \pi} a)$. On the right-hand side of Eqs. (46) and (47), the first terms $(\propto z)$ correspond to the Boltzmann gas, while the second terms $\left(\propto z^{2}\right)$ give the first quantum correction to the Boltzmann gas. The remaining terms proportional to $e^{x^{2}}$ and $e^{x^{2}} \operatorname{erfc}(x)$ are contributed from the bound state and the scattering states, respectively. The number of dimers $N^{\text {dimer }}$ is thus given from Eq. (47) by

$$
N^{\mathrm{dimer}}=2 \sqrt{2} \frac{V}{\lambda_{\mathrm{dB}}^{3}} e^{x^{2}} z^{2}
$$

The fugacity is determined from Eq. (47). In the hightemperature regime, where $\rho \lambda_{\mathrm{dB}}^{3} \ll 1$, we expand the fugacity up to the second order in $\rho \lambda_{\mathrm{dB}}^{3}$, obtaining

$$
z=\rho \lambda_{\mathrm{dB}}^{3}-\left[\frac{1}{2^{3 / 2}}+4 \sqrt{2}\left(e^{x^{2}}-\frac{e^{x^{2}} \operatorname{erfc}(x)}{2}\right)\right]\left(\rho \lambda_{\mathrm{dB}}^{3}\right)^{2} .
$$

Substituting this in Eq. (48), we obtain

$$
N^{\text {dimer }}=2 \sqrt{2} N\left(\rho \lambda_{\mathrm{dB}}^{3}\right) e^{x^{2}} .
$$

When the thermal energy dominates the binding energy of dimers, i.e., $x=\lambda_{\mathrm{dB}} /(\sqrt{2} \pi a) \ll 1$, the fraction of dimers is negligible and the system behaves like a classical gas of atoms.

On the other hand, when $x \gg 1$, it is possible for the number of dimers to be macroscopic. We may use this fact to calculate $f_{\mathrm{B}} e^{\beta \mathcal{H}_{1}} \otimes f_{\mathrm{B}} e^{\beta \mathcal{H}_{1}} U_{2}$. As shown in Eq. (C15) in Appendix $\mathrm{C}$, the contribution from the bound state reads

$$
\begin{aligned}
& \frac{1}{V} \operatorname{Tr}\left[f_{\mathrm{B}} f_{\mathrm{B}} e^{\beta\left(\mathcal{H}_{1}+\mathcal{H}_{1}\right)}|b\rangle\left\langle b\left|e^{-\beta \mathcal{H}_{2}}\right| b\right\rangle\langle b|\right] \\
& \quad=\frac{e^{x^{2}}}{\lambda_{\mathrm{dB}}^{3}}\left\{2 \sqrt{2} z^{2}+\frac{\pi^{-1 / 2}}{x^{3}} \sum_{n+m>2}^{\infty} \frac{z^{n+m}}{(2 n m-n-m)^{3 / 2}}\right\},
\end{aligned}
$$

where higher-order terms in the fugacity ( $z^{n}$ with $\left.n \geq 3\right)$ de-

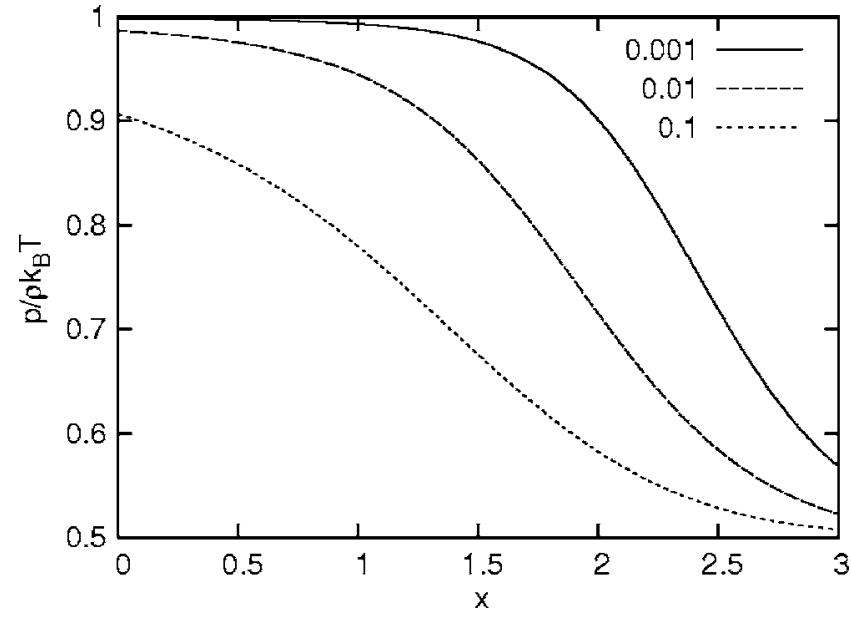

FIG. 2. Dependence of $p / \rho k_{\mathrm{B}} T$ on $x=\lambda_{\mathrm{dB}} / \sqrt{2 \pi} a$ for $\rho \lambda_{\mathrm{dB}}^{3}$ $=0.001,0.01,0.1$. With increasing $x$, the pressure decreases to about one half of that of the ideal atomic gas because of dimer formation.

scribe quantum exchange effects between dimer atoms and the surrounding atoms; however, their contributions are much smaller than the Maxwell-Boltzmann weight of dimers in the regime of $x \gg 1$.

When $k_{\mathrm{B}} T \ll\left|E_{\mathrm{b}}\right|$, the system behaves like a classical gas of dimers. Writing down the grand partition function with only the classical atomic term and the classical dimer term, we have

$$
\begin{gathered}
\beta p=\frac{1}{\lambda_{\mathrm{dB}}^{3}}\left[z+2 \sqrt{2}\left(e^{x^{2}}-\frac{e^{x^{2}} \operatorname{erfc}(x)}{2}\right) z^{2}\right], \\
z=\frac{\sqrt{1+8 \sqrt{2} e^{x^{2}}[2-\operatorname{erfc}(x)] \rho \lambda_{\mathrm{dB}}^{3}}-1}{4 \sqrt{2} e^{x^{2}}[2-\operatorname{erfc}(x)]} .
\end{gathered}
$$

It follows from these results that for $\rho \lambda_{\mathrm{dB}}^{3} \ll 1$ the pressure of the system decreases from $\rho k_{\mathrm{B}} T$ to $\rho k_{\mathrm{B}} T / 2$ with increasing $x$ as shown in Fig. 2. Since there is a bound state of the two-body problem, the pressure cannot reach $\rho k_{\mathrm{B}} T$. This fact continues to hold true at the unitarity limit of $a \rightarrow \infty$.

The universal thermodynamic relations for the pressure $p$ and the energy per volume $e$ are obtained from Eq. (46) up to the order of $\mathcal{O}\left(\left(\rho \lambda_{\mathrm{dB}}^{3}\right)^{2}\right)$ as

$$
\begin{gathered}
\frac{p}{\rho k_{\mathrm{B}} T}=1-\frac{9}{8} \sqrt{2}\left(\rho \lambda_{\mathrm{dB}}^{3}\right), \\
e=\frac{3}{2} \rho k_{\mathrm{B}} T\left[1-\frac{9}{8} \sqrt{2}\left(\rho \lambda_{\mathrm{dB}}^{3}\right)\right] .
\end{gathered}
$$

These expressions hold true for spinless bosons. The generalization of these results to cases with arbitrary spins is straightforward: for bosons with spin $J$, we have

$$
e=\frac{3}{2} \rho k_{\mathrm{B}} T\left[1-\frac{\sqrt{2}(J+1)+2^{-5 / 2}}{2 J+1} \rho \lambda_{\mathrm{dB}}^{3}\right]
$$

and for fermions with spin $J$, we have 


$$
e=\frac{3}{2} \rho k_{\mathrm{B}} T\left[1-\frac{\sqrt{2} J-2^{-5 / 2}}{2 J+1} \rho \lambda_{\mathrm{dB}}^{3}\right] .
$$

We also find that the relation $e=3 p / 2$ holds true up to the same order. For the special case of $J=1 / 2$, Eq. (57) reduces to the formula obtained in Ref. [19] using the Beth-Ulenbeck formula.

As long as we deal with many-body effects by using only the one-particle and two-particle clusters, the coefficients of the grand partition function (13) can be written as

$$
b_{l}=\frac{1}{\lambda_{\mathrm{dB}}^{3}} f_{l}(x),
$$

where $\lambda_{\mathrm{dB}}^{-3}$ is the contribution from the center-of-mass motion and $f_{l}$ is a dimensionless function of $x=\lambda_{\mathrm{dB}} /(\sqrt{2 \pi} a)$. If the function $f_{l}$ is regular with respect to $x$ and the fugacity is finite at the limit of $a \rightarrow \infty$, the relationship between the energy per volume and the pressure is $e=3 p / 2$. This is because the energy density is given by

$$
e=-\left(\frac{\partial}{\partial \beta} \frac{\ln \Xi}{V}\right)_{z}=\frac{3}{2} k_{\mathrm{B}} T \frac{\ln \Xi}{V}-\frac{k_{\mathrm{B}} T}{2} \sum_{l=1}^{\infty} z^{l} \lambda_{\mathrm{dB}}^{-3} x \frac{\partial}{\partial x} f_{l},
$$

where the first term on the right-hand side corresponds to the pressure given in Eq. (14), and the second term vanishes at the unitarity limit $(x \rightarrow 0)$.

\section{POSSIBLE COEXISTENCE OF ATOMIC AND DIMER BECS}

In the preceding sections, we have shown that thermal atoms, thermal dimers, and Bose condensed dimers can be described in terms of two dimensionless parameters $x$ $=\lambda_{\mathrm{dB}} / \sqrt{2 \pi} a$ and $\rho \lambda_{\mathrm{dB}}^{3}$. In Sec. IV, we assume that the binding energy is very strong $\left(\mu \leq E_{\mathrm{b}} / 2\right)$ and ignore the repulsive interaction between atoms. The dimers in our grand partition function behave as an ideal quantum gas since the diagrams of the repeated binary collisions do not give rise to the interactions between dimers. However, as the scattering length or the atomic density increases, the chemical potential should eventually overcome the binding energy and then our approximation would become invalidated. This possibility will be discussed in a future publication.

Here we discuss a coexistence of atomic and dimer BECs within our grand partition function. This is possible if the first excited state of the dimer BEC lies above the atomic BEC state and if the thermal energy is large enough to populate the atomic BEC state.

Let us estimate the number of states $\mathcal{N}$ that lie below the zero-energy state of atoms. Assuming that the Hamiltonian supports only one bound state, we can focus on the centerof-mass motion of dimers. Hence $\mathcal{N}$ can be estimated in the same way as for free particles, giving

$$
\mathcal{N}=\frac{V}{(2 \pi \hbar)^{3}} \frac{4 \pi}{3}\left|4 m E_{\mathrm{b}}\right|^{3 / 2} \sim \frac{N}{\rho a^{3}} .
$$

Thus the atomic BEC state may exist for $x<1$ and $\rho a^{3}>1$. In fact, when the energy of the first excited state of the mo- lecular BEC, $E_{\mathrm{b}}+\Delta$, is positive and the thermal energy is large enough to excite the zero energy state, the occupation number of the atomic BEC state could be macroscopic at low temperatures such that $x<1$ and $k_{\mathrm{B}} T<\Delta$.

In a bulk system, the excitation spectrum of dimers is continuous, so the atomic BEC state can hardly be occupied by a large number of particles. However, in a trapped system, the BEC state can be populated appreciably because the excited states are distributed in a discrete manner. For instance, since $\Delta=\hbar \omega$ in a harmonic trap, $E_{\mathrm{b}}+\Delta>0$ implies $a>d_{\mathrm{ho}}$ where the $d_{\mathrm{ho}}=\sqrt{\hbar / m \omega}$ is the size of the ground-state wave function of an ideal BEC in a harmonic trap. When the energy of the first excited state of the dimer is higher than the atomic BEC state, the atomic BEC state is considered to be in the first excited state of the system. Hence, if $\left|E_{\mathrm{b}}\right|<k_{\mathrm{B}} T$ $<\Delta$, the thermal fluctuations do not significantly excite the excited states of the dimers but do populate the zero-energy state of atoms. This implies that if the condition $a>\lambda_{\mathrm{dB}}$ $>d_{\mathrm{ho}}$ is met, an atomic BEC can coexist with a dimer BEC.

\section{SUMMARY AND CONCLUSIONS}

In this paper, we have applied the Lee-Yang cluster expansion method to investigate quantum-statistical properties of a mixture of atoms and dimers. We consider a system of bosons and that of fermions with arbitrary spins, interacting via $s$-wave scattering.

We truncate the cluster expansion by taking only one-particle and two-particle clusters. The one-particle cluster describes the quantum exchange effect and the ring diagram of the one-particle clusters shown in Fig. 1(a) leads to Bose-Einstein condensation (BEC) of atoms [20]. If the two-body Hamiltonian supports the bound state, the ring diagram of repeated collisions via two-particle clusters is shown to lead to Bose-Einstein condensation of dimers. We have taken into account the renormalization of the one-particle cluster due to the exchange of dimer atoms with surrounding atoms as shown in Fig. 1(d). Thus our theory takes into account the interconversion of atoms between dimers and surrounding atoms.

When the scattering length $a$ is much larger than the effective range $l_{\text {eff }}$ of the interaction, the grand partition function and the equation of state depend only on the thermal de Broglie length $\lambda_{\mathrm{dB}}$ and $a$. The large ratio of $x=\lambda_{\mathrm{dB}} /(\sqrt{2 \pi} a)$ implies weak scattering and strongly bound dimers if they exist. On the other hand, the small ratio is achieved in the universality limit near the Feshbach resonance. We evaluated the number of dimers in the high-temperature regime as a function of $x$, and found that with increasing $x$ the number of dimers grows and the pressure of the system decreases to about one half due to formation of dimers. We also found the pressure and the energy in the universality limit of $x \rightarrow 0$ for systems of an arbitrary spin up to the second order in the number density.

When we consider the interacting bose system that has a bound state, it is of interest to ask whether the BEC of atoms and that of dimers coexist. Our grand partition function suggests that in the bulk system the dimer BEC is dominant because of a finite binding energy and the continuous exci- 
tation spectrum of dimers. However, in a trapped system, the coexistence of atomic and dimers BECs is implied when the scattering length is larger than both the thermal de Broglie length and the system size.

In this paper, in the grand partition function we take only the ring diagrams of one-particle and two-particle clusters and neglect the higher-order effects. In particular, the interaction between dimers is not included. It is not clear, however, whether the ground-state energy of dimers is lower than that of atoms in the presence of repulsive interaction between dimers. This subject merits further study.

\section{ACKNOWLEDGMENTS}

This work was supported by a Grant-in-Aid for Science Research (Grant No. 17071005) and a 21st Century COE program at Tokyo Tech "Nanometer-Scale Quantum Physics" from the Ministry of Education, Culture, Sports, Science and Technology of Japan. M.U. acknowledges support by a CREST program of the JST.

\section{APPENDIX A: DERIVATION OF CLUSTER EXPANSION}

To make the paper self-contained, we outline a derivation of the cumulant expansion formula of the grand partition function applicable to both classical and quantum systems. Using the Boltzmann factors $W_{n}, W_{n}^{\mathrm{S}}$, and $W_{n}^{\mathrm{A}}$ in Eqs. (3), (7), and (8), respectively, we evaluate the corresponding partition functions, $Q_{N}$, for an $N$ particle system

$$
\begin{aligned}
Q_{n}^{\mathrm{cl}} & =\frac{1}{N !} \sum_{1, \ldots, N}\left\langle 1, \ldots, N\left|W_{N}\right| 1, \ldots, N\right\rangle, \\
Q_{N}^{\mathrm{S}, \mathrm{A}} & =\frac{1}{N !} \sum_{1, \ldots, N}\left\langle 1, \ldots, N\left|W_{N}^{\mathrm{S}, \mathrm{A}}\right| 1, \ldots, N\right\rangle .
\end{aligned}
$$

Since the procedure for the classical system applies equally to the quantum system, we only show the derivation for the classical system. The grand partition function is defined by

$$
\Xi=\sum_{N=0}^{\infty} z^{N} Q_{N},
$$

where $z \equiv e^{\beta \mu}$ is the fugacity. We then decompose $W_{N}$ into $j$-particle clusters $U_{j}$, and regroup the grand partition function as follows:

$$
\begin{aligned}
\Xi= & \sum_{N=0}^{\infty} \sum_{\left\{m_{j}\right\}} \frac{z^{N}}{N !} \prod_{j=1}^{N} \frac{N !}{\prod_{j}(j !)^{m_{j} m_{j} !}} \\
& \times\left(\sum_{1, \ldots, j}\left\langle 1, \ldots, j\left|U_{j}\right| 1, \ldots, j\right\rangle\right)^{m_{j}} \\
= & \prod_{j=1}^{\infty} \sum_{m_{j}=0}^{\infty} \frac{1}{m_{j} !}\left(\frac{z}{j !} \sum_{1, \ldots, j}\left\langle 1, \ldots, j\left|U_{j}\right| 1, \ldots, j\right\rangle\right)^{m_{j}} \\
= & \exp \left(V \sum_{j=1}^{\infty} z^{j} b_{j}\right),
\end{aligned}
$$

where the coefficient $b_{j}$ is defined as

$$
b_{j} \equiv \frac{1}{V j !} \sum_{1, \ldots, j}\left\langle 1, \ldots, j\left|U_{j}\right| 1, \ldots, j\right\rangle .
$$

In Eq. (A4), $\Sigma_{\left\{m_{j}\right\}}$ implies summation over all possible sets $\left\{m_{j}\right\}$ that satisfy $\sum_{j=1}^{N} j m_{j}=N$, and the factor $N ! /\left[\Pi_{j}(j !)^{m_{j}} m_{j} !\right]$ is the number of the particle configurations for a given $\left\{m_{j}\right\}$. We can obtain the corresponding formulas for the quantum system by replacing $W_{N}$ and $U_{j}$ with $W_{N}^{\mathrm{S}, \mathrm{A}}$ and $U_{j}^{\mathrm{S}, \mathrm{A}}$, respectively.

\section{APPENDIX B: GRAND PARTITION FUNCTION FOR AN ARBITRARY SPIN SYSTEM}

We derive the grand partition function for a system of fermions or bosons with spin $J$. The two-body interaction of the system is assumed to be independent of the spin. We first consider the case of fermions.

Let us evaluate the number of topologically distinct diagrams, $K_{2 n}^{J}$, with respect to the spin configuration for a sigle loop composed of $n$ two-particle clusters involving $2 n$ independent fermions with spin $J$. We must take into account the spin degrees of freedom in performing the trace operation. Let the ket vector of the spin state be $\left|\sigma_{i}, \sigma_{j}\right\rangle$, where $i$ and $j$ distinguish particles. Since there are two states, $\left\langle\sigma_{i}, \sigma_{j}\right|$ and $\left\langle\sigma_{j}, \sigma_{i}\right|$, in the trace operation for each pair of particles, the contribution to the grand partition function reads

$$
\sum_{\{\sigma\} \text { loop }} \prod_{i}\left(\left\langle\sigma_{i}, \sigma_{j}\left|-\left\langle\sigma_{j}, \sigma_{i}\right|\right) U_{2} \mid \sigma_{k}, \sigma_{l}\right\rangle,\right.
$$

where we assumed that $i<j$ and $k<l$, and the minus sign in Eq. (B1) arises from the Fermi statistics. We interpret Eq. (B1) as a series of transitions of two particles from the ket vector to the bra vector through $U_{2}$. We shall refer to $\left(\left\langle\sigma_{i}, \sigma_{j}\left|-\left\langle\sigma_{j}, \sigma_{i}\right|\right) U_{2} \mid \sigma_{k}, \sigma_{l}\right\rangle\right.$ as portion. If the $i$ th particle and the $j$ th particle have the same spin, the contribution (B1) vanishes. Thus we only consider the situation in which $\sigma_{i}$ is different from $\sigma_{j}$. By considering transitions of a pair of particles from a ket to a bra, we identify nonvanishing spin alignments. We first consider the following portion:

$$
\sum_{\sigma_{3}, \sigma_{4}}\left|\sigma_{3}, \sigma_{4}\right\rangle\left(\left\langle\sigma_{3}, \sigma_{4}\left|-\left\langle\sigma_{4}, \sigma_{3}\right|\right) U_{2} \mid \sigma_{1}, \sigma_{2}\right\rangle\right.
$$

Suppose that $\sigma_{1}=j, \sigma_{2}=j^{\prime}$, and $j \neq j^{\prime}$. Then, nonzero contributions arise from the "direct" set $\left(\sigma_{3}=j\right.$ and $\left.\sigma_{4}=j^{\prime}\right)$ and the "exchange" set $\left(\sigma_{3}=j^{\prime}, \sigma_{4}=j\right)$, and if the exchange set is chosen, the minus sign appears as in the second term in Eq. (B2). By the same token, in the next portion of $\left|\sigma_{3}, \sigma_{4}\right\rangle$, only $\left|j, j^{\prime}\right\rangle$ and $\left|j^{\prime}, j\right\rangle$ give nonzero contributions. The crucial constraint is to form loop; for this loop to give a nonvanishing contribution we must have

$$
\left(\left\langle\sigma_{1}, \sigma_{2}\left|-\left\langle\sigma_{2}, \sigma_{1}\right|\right) U_{2} \mid \sigma_{2 n}, \sigma_{2 n-1}\right\rangle \neq 0 .\right.
$$

We can thus categorize nonvanishing transitions of a pair of particles from one $U_{2}$ to another into three possibilities: (i) All intermediate states are direct sets. In this case $\left|\sigma_{2 n}, \sigma_{2 n-1}\right\rangle$ is, of course, equal to the direct set $\left|j, j^{\prime}\right\rangle$. (ii) Intermediate 
states include an odd number of exchange sets. In this case $\left|\sigma_{2 n}, \sigma_{2 n-1}\right\rangle$ is equal to $\left|j^{\prime}, j\right\rangle$. Since the minus signs also appear at odd times, this term carries the minus sign. (iii) Intermediate states include an even number of exchange sets. In this case $\left|\sigma_{2 n}, \sigma_{2 n-1}\right\rangle$ is equal to $\left|j, j^{\prime}\right\rangle$. This term carries the plus sign. Finally, the number of choices of a pair of $j$ and $j^{\prime}$ is $(2 J+1)^{2}-(2 J+1)$. Therefore,

$$
K_{2 n}^{J}=2^{n-1}\left[(2 J+1)^{2}-(2 J+1)\right]=2^{n} J(2 J+1) .
$$

We next consider a situation in which $m$ particles participate in one-particle clusters between the two-particle clusters. Let $C_{2 n+m}^{J}$ be the number of particle configurations for this situation. When there are $2 n$ particles and $n U_{2}$ clusters, i.e., $m=0$, the sign $\operatorname{sgn} \mathfrak{P}$ of the nonvanishing contribution is always positive. The quantum exchange of atoms occurs on single-particle lines between two-particle clusters. Let us consider a partial representation of a permutation such as

$$
\left\langle i\left|U_{1}\right| j\right\rangle\left\langle j\left|U_{1}\right| k\right\rangle\left\langle k, l\left|U_{2}\right| n, m\right\rangle .
$$

The corresponding permutation reads

$$
\left(\begin{array}{cccc}
j & k & n & m \\
i & j & k & l
\end{array}\right)
$$

By performing the trace for the $j$ particle, we can eliminate the state of $j$ from Eq. (B5), giving

$$
\left\langle i\left|U_{1}^{2}\right| k\right\rangle\left\langle k, l\left|U_{2}\right| n, m\right\rangle \text {. }
$$

Accordingly, the representation of the permutation is changed into

$$
\left(\begin{array}{cccc}
j & k & n & m \\
j & i & k & l
\end{array}\right)
$$

Comparing Eqs. (B6) and (B8), we find that the trace operation over the $j$ particle amounts to the transposition of $i$ and $j$. Furthermore, if the trace for the $k$ particle is carried out, Eq. (B5) becomes

$$
\left(\left\langle i\left|U_{1}^{2} \otimes\langle l|\right) U_{2} \mid n, m\right\rangle .\right.
$$

The corresponding representation of the permutation reads

$$
\left(\begin{array}{cccc}
j & k & n & m \\
j & k & i & l
\end{array}\right)
$$

Comparing Eqs. (B8) and (B10), we find that the trace operation over the $k$ particle amounts to the transposition of $i$ and $k$. Thus each trace operation can be represented by one permutation which yields a factor of -1 , and therefore tracing over $m$ atoms adds a factor of $(-1)^{m}$. The total contribution from the sign of the permutation is $\operatorname{sgn} \mathfrak{P}=(-1)^{m}$. Thus we can calculate $C_{2 n+m}^{J}$ using $K_{2 n}^{J}$ as

$$
C_{2 n+m}^{J}=(-1)^{m} K_{2 n}^{J} \frac{2 n+m}{C_{2} \times \cdots \times{ }_{2+m} C_{2}} m !
$$

We can use this result to find the contribution to the grand partition function as a partial sum

$$
\begin{aligned}
\sum_{n=1}^{\infty} \sum_{m=0}^{\infty} \sum_{m=m_{1}+\cdots+m_{2 n}} \frac{z^{2 n+m}}{(2 n+m) !} C_{2 n+m}^{J} \operatorname{Tr}_{\mathbf{k}_{1}, \ldots, \mathbf{k}_{2 n}}\left\{\left(U_{1}^{m_{1}} \otimes U_{1}^{m_{2}} U_{2}\right) \times \cdots \times\left(U_{1}^{m_{2 n-1}} \otimes U_{1}^{m_{2 n}} U_{2}\right)\right\} \\
=J(2 J+1) \sum_{n=1}^{\infty} \frac{z^{2 n}}{n} \operatorname{Tr}_{\mathbf{k}_{1}, \ldots, \mathbf{k}_{2 n}}\left[\left(\sum_{m_{1}=0}^{\infty}(-z)^{m_{1}} U_{1}^{m_{1}} \otimes \sum_{m_{2}=0}^{\infty}(-z)^{m_{2}} U_{1}^{m_{2}} \cdot U_{2}\right)\right. \\
\left.\quad \times \cdots \times\left(\sum_{m_{2 n-1}=0}^{\infty}(-z)^{m_{2 n-1}} U_{1}^{m_{2 n-1}} \otimes \sum_{m_{2 n}=0}^{\infty}(-z)^{m_{2 n}} U_{1}^{m_{2 n}} U_{2}\right)\right] \\
=J(2 J+1) \sum_{n=1}^{\infty} \frac{z^{2 n}}{n} \operatorname{Tr}_{\mathbf{k}_{1}, \mathbf{k}_{2}}\left(\frac{1}{1+z U_{1}} \otimes \frac{1}{1+z U_{1}} U_{2}\right)^{n}=-J(2 J+1) \operatorname{Tr}_{\mathbf{k}_{1}, \mathbf{k}_{2}} \ln \left[1-f_{\mathrm{F}}\left(\mathcal{H}_{1}\right) \otimes f_{\mathrm{F}}\left(\mathcal{H}_{1}\right)(\mathcal{S}-1)\right],
\end{aligned}
$$

where $f_{\mathrm{F}}\left(\mathcal{H}_{1}\right)=\left[\exp \left\{\beta\left(\mathcal{H}_{1}-\mu\right)\right\}+1\right]^{-1}$ is the Fermi-Dirac distribution operation, and $\operatorname{Tr}_{\mathbf{k}} \ldots$ implies the trace operation over $\mathbf{k}$ only, i.e.

$$
\operatorname{Tr}_{\mathbf{k}_{1}, \ldots, \mathbf{k}_{n}} \cdots=\left(\prod_{i=1}^{n} \int d \mathbf{k}_{i}\right)\left\langle\mathbf{k}_{1}, \ldots, \mathbf{k}_{n}|\ldots| \mathbf{k}_{1}, \ldots, \mathbf{k}_{n}\right\rangle .
$$

For the case of bosons, we consider

$$
\sum_{\sigma_{3}, \sigma_{4}}\left|\sigma_{3}, \sigma_{4}\right\rangle\left(\left\langle\sigma_{3}, \sigma_{4}\left|+\left\langle\sigma_{4}, \sigma_{3}\right|\right) U_{2} \mid \sigma_{1}, \sigma_{2}\right\rangle,\right.
$$

where the plus sign corresponds to Bose statistics. In a manner similar to Fermi statistics, we can calculate $K_{2 n}^{J}$ and $C_{2 n+m}^{J}$ as

$$
K_{2 n}^{J}=2^{n-1}\left[(2 J+1)^{2}+(2 J+1)\right]=2^{n-1}(2 J+2)(2 J+1),
$$




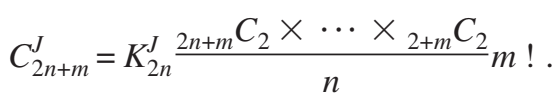

The difference from the case of fermions is that the diagonal spin states $\left|\sigma_{3}, \sigma_{4}\right\rangle=|j, j\rangle$ do not vanish. The partition function of a Bose system is obtained by the replacement of the fugacity, $z$, in the fermions system with $-z$ in the Bose system

$$
\frac{1}{1+z U_{1}} \rightarrow \frac{1}{1-z U_{1}}
$$

Using the coefficient of $C_{2 l+n}^{J}$, we can explicitly calculate the correction of the grand partition function in a manner similar to Eq. (B12). We thus obtain Eq. (34).

\section{APPENDIX C: CALCULATION OF $\operatorname{Tr}\left\{\left(f_{\mathrm{B}} \otimes f_{\mathrm{B}}\right)(\mathcal{S}-1)\right\}$}

In this appendix, we evaluate the trace of $\left(f_{\mathrm{B}} \otimes f_{\mathrm{B}}\right)(\mathcal{S}$ $-1)$ and discuss the associated quantum exchange effect. First, we solve the two-body problem defined by the Hamiltonian,

$$
\mathcal{H}_{2}=\frac{\mathbf{p}_{1}^{2}}{2 m}+\frac{\mathbf{p}_{2}^{2}}{2 m}+\mathcal{V}_{\text {pseudo }}\left(\mathbf{r}_{2}-\mathbf{r}_{1}\right)
$$

where $\mathcal{V}_{\text {pseudo }}$ is given in Eq. (45). The center-of-mass motion is easily solved. Let us consider the relative motion within the $s$-wave approximation. The boundary condition near the origin of the relative coordinate is written for arbitrary wave function $\psi$ as

$$
-\int_{\partial V_{\epsilon}} \epsilon^{2} \frac{\partial \psi}{\partial r} d \Omega+\left.4 \pi a \frac{\partial}{\partial r}(r \psi)\right|_{r=0}=0
$$

where $d \Omega$ denotes integration over the solid angle and $\epsilon$ is an infintesimal positive number. The Hamiltonian (C1) supports one bound state $\psi_{\mathrm{b}}$ and continuous scattering states $\psi_{\mathbf{k}_{s}}$ as

$$
\begin{aligned}
& E_{\mathrm{b}}=-\frac{\hbar^{2}}{m a^{2}}, \\
& \psi_{\mathrm{b}}(\mathbf{r})=\frac{1}{\sqrt{2 \pi a}} \frac{e^{-r / a}}{r} ;
\end{aligned}
$$

$$
\begin{aligned}
& E_{s}=\frac{\hbar^{2} k_{s}^{2}}{m}, \\
& \psi_{k_{s}}(\mathbf{r})=\sqrt{\frac{2}{\pi\left(1+\left(k_{s} a\right)^{2}\right)}} \frac{\sin k_{s} r-k_{s} a \cos k_{s} r}{r} Y_{0,0} .
\end{aligned}
$$

We use these results to evaluate the two-particle cluster $\left\langle\mathbf{r}_{1}^{\prime}, \mathbf{r}_{2}^{\prime}\left|U_{2}\right| \mathbf{r}_{1}, \mathbf{r}_{2}\right\rangle$ :

$$
\begin{aligned}
& \left\langle\mathbf{r}_{1}^{\prime}, \mathbf{r}_{2}^{\prime}\left|U_{2}\right| \mathbf{r}_{1}, \mathbf{r}_{2}\right\rangle=\frac{2 \sqrt{2}}{\lambda_{\mathrm{dB}}^{3}} e^{-m\left(\mathbf{R}-\mathbf{R}^{\prime}\right)^{2} / \hbar^{2} \beta}\left\{\psi_{\mathrm{b}}(r) \psi_{\mathrm{b}}\left(r^{\prime}\right) e^{-\beta E_{\mathrm{b}}}+\int_{0}^{\infty} d k e^{-\beta \hbar^{2} k^{2} / m}\left[\psi_{k}(r) \psi_{k}\left(r^{\prime}\right)-\frac{\sin k r \sin k r^{\prime}}{2 \pi^{2} r r^{\prime}}\right]\right\} \\
& =\frac{2 \sqrt{2}}{\lambda_{\mathrm{dB}}^{3}} e^{-m\left(\mathbf{R}-\mathbf{R}^{\prime}\right)^{2} / \hbar^{2} \beta}\left\{\psi_{\mathrm{b}}(r) \psi_{\mathrm{b}}\left(r^{\prime}\right) e^{-\beta E_{\mathrm{b}}}+\frac{1}{2 \pi^{2} r r^{\prime}}\left[\sqrt{\frac{\pi m}{4 \hbar^{2} \beta}} e^{-\frac{m\left(r+r^{\prime}\right)^{2}}{4 \hbar^{2} \beta}}-\frac{\pi}{2 a} e^{x^{2}} e^{-\left(r+r^{\prime}\right) / a} \operatorname{erfc}\left(x-\frac{r+r^{\prime}}{2 a x}\right)\right]\right\},
\end{aligned}
$$

where $\mathbf{R}$ and $\mathbf{r}$ denote the center-of-mass coordinate and the relative coordinate, respectively, and $x=\lambda_{\mathrm{dB}} /(\sqrt{2 \pi} a)$. The function of $\operatorname{erfc}(x)$ is the complete error function defined by $1-\operatorname{erf}(x)$, and the error function is defined as

$$
\operatorname{erf}(x)=\frac{2}{\sqrt{\pi}} \int_{0}^{x} e^{-t^{2}} d t .
$$

The corresponding wave vector representation is given by

$$
\begin{aligned}
\left\langle\mathbf{k}_{1}^{\prime}, \mathbf{k}_{2}^{\prime}\left|U_{2}\right| \mathbf{k}_{1}, \mathbf{k}_{2}\right\rangle= & \delta^{3}\left(\mathbf{k}_{1}^{\prime}+\mathbf{k}_{2}^{\prime}-\mathbf{k}_{1}-\mathbf{k}_{2}\right) e^{-\hbar^{2} \beta \mathbf{K}^{2} / 4 m} \\
& \times\left\{\frac{a^{3}}{\pi^{2}} \frac{e^{-\beta E_{\mathrm{b}}}}{\left[1+(k a)^{2}\right]\left[1+\left(k^{\prime} a\right)^{2}\right]}-\frac{4}{\pi} \frac{1}{k^{2}-k^{\prime 2}} \int d \xi\left[\frac{\sin 2 k \xi}{k}-\frac{\sin 2 k^{\prime} \xi}{k^{\prime}}\right] f_{s}(\xi)\right\},
\end{aligned}
$$

where

$$
f_{s}(\xi)=\frac{1}{2 \pi^{2}}\left[\frac{\sqrt{\pi}}{2} \frac{e^{-(\xi / a x)^{2}}}{a x}-\frac{\pi}{2 a} e^{x^{2}} e^{2 \xi / a} \operatorname{erfc}\left(x-\frac{\xi}{a x}\right)\right]
$$

and the $\mathbf{K}$ and $\mathbf{k}$ are the center-of-mass momentum and the relative momentum, respectively. Performing the integration, we obtain 


$$
\begin{aligned}
\left\langle\mathbf{k}_{1}^{\prime}, \mathbf{k}_{2}^{\prime}\left|U_{2}\right| \mathbf{k}_{1}, \mathbf{k}_{2}\right\rangle= & \delta^{3}\left(\mathbf{K}-\mathbf{K}^{\prime}\right) e^{-\beta \hbar^{2} K^{2} / 4 m}\left\{\frac{a^{3}}{\pi^{2}} \frac{e^{-\beta E_{\mathrm{b}}}}{\left[1+(k a)^{2}\right]\left[1+\left(k^{\prime} a\right)^{2}\right]}-\frac{1}{2 \pi^{2}} \frac{1}{k+k^{\prime}} \frac{1}{\left(k-k^{\prime}\right)}\left[\frac { 1 } { k } \left(\frac{(k a)^{2}}{1+(k a)^{2}} e^{-(k a x)^{2}} \operatorname{erfi}(k a x)\right.\right.\right. \\
& \left.-\frac{k a}{1+(k a)^{2}} e^{x^{2}} \operatorname{erfc}(x)-\frac{k a}{1+(k a)^{2}} e^{-(k a x)^{2}}\right)-\frac{1}{k^{\prime}}\left(\frac{\left(k^{\prime} a\right)^{2}}{1+\left(k^{\prime} a\right)^{2}} e^{-\left(k^{\prime} a x\right)^{2}} \operatorname{erfi}\left(k^{\prime} a x\right)-\frac{k^{\prime} a}{1+\left(k^{\prime} a\right)^{2}} e^{x^{2}} \operatorname{erfc}(x)\right. \\
& \left.\left.\left.-\frac{k^{\prime} a}{1+\left(k^{\prime} a\right)^{2}} e^{-\left(k^{\prime} a x\right)^{2}}\right)\right]\right\},
\end{aligned}
$$

where $\operatorname{erfi}(x)$ is the imaginary error function defined as erfi $(x) \equiv-i \operatorname{erf}(i x)$.

Finally, we evaluate the trace of $\left(f_{\mathrm{B}} \otimes f_{\mathrm{B}}\right)(\mathcal{S}-1)$ using Eq. (38). When we calculate the trace in terms of the wave vector representation, the $\delta$ function in the two-particle cluster $U_{2}$ is replaced with the factor $\frac{1}{(2 \pi)^{3}}$. Thus

$$
\begin{aligned}
\frac{1}{V} \operatorname{Tr}\left\{\left(f_{\mathrm{B}} \otimes f_{\mathrm{B}}\right)(\mathcal{S}-1)\right\} & =\frac{1}{(2 \pi)^{3}} \int d \mathbf{k}_{1} d \mathbf{k}_{2} \sum_{n_{1}=1}^{\infty} \sum_{n_{2}=1}^{\infty} z^{n_{1}+n_{2}} e^{-n_{1} \hbar^{2} \beta k_{1}^{2} / 2 m} e^{-n_{2} \beta \hbar^{2} k_{2}^{2} / 2 m} u_{2}(k) e^{\beta\left[\hbar^{2} / 4 m K^{2}+\left(\hbar^{2} / m\right) k^{2}\right]} \\
& =\frac{1}{(2 \pi)^{3}} \sum_{n_{1}, n_{2}} \int d \tilde{\mathbf{K}} d \mathbf{k} z^{n_{1}+n_{2}} e^{-\beta\left(n_{1}+n_{2}\right) \hbar^{2} \tilde{K}^{2} / 8 m} e^{-2 \beta \alpha \hbar^{2} k^{2} / m} u_{2}(k) \\
& =\sum_{n_{1}, n_{2}} \frac{1}{\lambda_{\mathrm{dB}}^{3}} \frac{z^{n_{1}+n_{2}}}{\left(n_{1}+n_{2}\right)^{3 / 2}}\left(C_{\mathrm{b}}+C_{\mathrm{s}}\right),
\end{aligned}
$$

where $\tilde{\mathbf{K}}=2 \frac{n_{1} \mathbf{k}_{1}+n_{2} \mathbf{k}_{2}}{n_{1}+n_{2}}, \alpha=\frac{n_{1} n_{2}}{n_{1}+n_{2}}$,

$$
\begin{aligned}
u_{2}(k)= & \frac{a^{3}}{\pi^{2}} \frac{e^{\beta \hbar^{2} k^{2} / m}}{\left[1+(k a)^{2}\right]^{2}} e^{x^{2}} \\
& -e^{\beta \hbar^{2} k^{2} / m} \frac{2}{\pi} \frac{1}{k} \frac{\partial}{\partial k} \int_{0}^{\infty} d \xi f_{s}(\xi) \frac{\sin 2 k \xi}{k},
\end{aligned}
$$

and the coefficients $C_{\mathrm{b}}$ and $C_{\mathrm{s}}$ describe the contribution from the bound state of the first term of $u_{2}$ and that from the scattering states of the same term, respectively. By exchanging the order of integrations over $k$ and $\xi$, we obtain

$$
\begin{aligned}
& C_{\mathrm{b}}=8\left\{-2 \sqrt{\frac{2 \alpha-1}{\pi}} x+\left[1+2(2 \alpha-1) x^{2}\right]\right. \\
& \left.\times e^{(2 \alpha-1) x^{2}} \operatorname{erfc}(\sqrt{2 \alpha-1} x)\right\} e^{x^{2}}, \\
& C_{\mathrm{s}}=\frac{8}{\pi}\left\{\arctan \frac{1}{\sqrt{2 \alpha-1}}-\frac{1}{\sqrt{2 \alpha-1}-\frac{1}{\sqrt{2 \alpha-1}}}\right\} \\
& -8 e^{x^{2}} \int_{0}^{\infty} d t e^{-2 t} \operatorname{erfc}\left(x-\frac{t}{x}\right) \operatorname{erf}\left(\frac{t}{x \sqrt{2 \alpha-1}}\right) \\
& +\frac{16}{\sqrt{\pi}} \frac{1}{x \sqrt{2 \alpha-1}} e^{x^{2}} \int_{0}^{\infty} d t t \operatorname{erfc}\left(x-\frac{t}{x}\right) e^{-2 t-\left[t^{2} / x^{2}(2 \alpha-1)\right]} .
\end{aligned}
$$

For sufficiently small $x$, we obtain

$$
\begin{aligned}
& \frac{1}{V} \operatorname{Tr}\left\{f_{\mathrm{B}} \otimes f_{\mathrm{B}}(\mathcal{S}-1)\right\} \\
& \cong \frac{1}{\lambda_{\mathrm{dB}}^{3} \sum_{1}, n_{2}} \frac{z^{n_{1}+n_{2}}}{\left(n_{1}+n_{2}\right)^{3 / 2}} 8\left\{1-4 \sqrt{\left.\frac{2 \alpha-1}{\pi} x\right\}}\right. \\
& \quad+\frac{1}{\lambda_{\mathrm{dB}}^{3}} \sum_{n_{1}, n_{2}} \frac{z^{n_{1}+n_{2}}}{\left(n_{1}+n_{2}\right)^{3 / 2}}\left[\frac { 8 } { \pi } \left\{\arctan \frac{1}{\sqrt{2 \alpha-1}}\right.\right. \\
& \left.\quad-\frac{1}{\sqrt{2 \alpha-1}-\frac{1}{\sqrt{2 \alpha-1}}}\right\}+8\left\{-1+6 \sqrt{\frac{2 \alpha-1}{\pi}} x\right\},
\end{aligned}
$$

where we ignore high-order terims in $\sqrt{2 \alpha-1} x$. The limit $x \rightarrow 0$ corresponds to the unitarity limit. In addition, the energy of the bound state goes to zero. The fact that the coefficients of higher-order terms in the fugacity remain nonvanishing at the limit of $x \rightarrow \infty$ implies that the quantum exhange effect between dimers and surrounding atoms is important in the same limit.

On the other hand, the expansion of the partition function for large $x$, which corresponds to strongly bound dimers, is given by 


$$
\frac{1}{V} \operatorname{Tr}\left\{f_{\mathrm{B}} \otimes f_{\mathrm{B}}(\mathcal{S}-1)\right\} \cong \frac{1}{\lambda_{\mathrm{dB}}^{3}}\left\{2 \sqrt{2} z^{2}+2 \sqrt{2} \pi\left(\frac{a}{\lambda_{\mathrm{dB}}}\right)^{3} \sum_{n_{1}+n_{2}>3} \frac{z^{n_{1}+n_{2}}}{\left(2 n_{1} n_{2}-n_{1}-n_{2}\right)^{3 / 2}}\right\} e^{x^{2}}-\frac{1}{\lambda_{\mathrm{dB}}^{3}}\left(\frac{2 a}{\lambda_{\mathrm{dB}}}\right) \sum_{n_{1}, n_{2}} \frac{1}{n_{1}^{3 / 2} n_{2}^{3 / 2}} z^{n_{1}+n_{2}} .
$$

Since the factor contributed from the bound state, $e^{x^{2}}$, is very large in this limit, the scattering effect is negligible. Furthermore, the quantum exhange effect is not important even if the fugacity is close to 1 . This implies that a composite particle within which two particles are strongly bound together behave as one Bose particle.

[1] S. Inouye, M. R. Andrews, J. Stenger, H.-J. Miesner, D. M. Stamper-Kurn, and W. Ketterle, Nature (London) 392, 151 (1998).

[2] S. L. Cornish, N. R. Claussen, J. L. Roberts, E. A. Cornell, and C. E. Wieman, Phys. Rev. Lett. 85, 1795 (2000).

[3] J. Herbig, T. Kraemer, M. Mark, T. Weber, C. Chin, H.-C. Nägerl, and R. Grimm, Science 301, 1510 (2003)

[4] K. Xu, T. Mukaiyama, J. R. Abo-Shaeer, J. K. Chin, D. E. Miller, and W. Ketterle, Phys. Rev. Lett., 91, 210402 (2003).

[5] S. Dürr, T. Volz, A. Marte, and G. Rempe, Phys. Rev. Lett. 92, 020406 (2004).

[6] C. A. Regal, C. Ticknor, J. L. Bohn, and D. S. Jin, Nature (London) 424, 47 (2003).

[7] K. E. Strecker, G. B. Partridge, and R. G. Hulet, Phys. Rev. Lett. 91, 080406 (2003).

[8] J. Cubizolles, T. Bourdel, S. J. J. M. F. Kokkelmans, G. V. Shlyapnikov, and C. Salomon, Phys. Rev. Lett. 91, 240401 (2003).

[9] S. Jochim, M. Bartenstein, A. Altmeyer, G. Hendl, C. Chin, J. H. Denschlag, and R. Grimm, Phys. Rev. Lett. 91, 240402
(2003).

[10] S. Jochim, M. Bartenstein, A. Altmeyer, G. Hendl, S. Riedl, C. Chin, J. H. Denschlag, and R. Grimm, Science 302, 2101 (2003).

[11] M. W. Zwierlein, C. A. Stan, C. H. Schunck, S. M. F. Raupach, S. Gupta, Z. Hadzibabic, and W. Ketterle, Phys. Rev. Lett. 91, 250401 (2003).

[12] C. A. Regal, M. Greiner, and D. S. Jin, Phys. Rev. Lett. 92, 040403 (2004)

[13] T. D. Lee and C. N. Yang, Phys. Rev. 113, 1165 (1959); 113, 1406 (1959); 117, 12 (1960); 11722 (1960); 117897 (1960).

[14] H. D. Ursell, Proc. Cambridge Philos. Soc. 23, 685 (1927).

[15] J. E. Mayer, J. Chem. Phys. 5, 67 (1937); J. E. Mayer, et al., J. Chem. Phys. 5, 74 (1937); 6, 87 (1938); 6, 101 (1938).

[16] J. J. Sakurai, Modern Quantum Mechanics (Benjamin, 1985).

[17] K. Wódkiewicz, Phys. Rev. A 43, 68 (1990).

[18] K. Huang, Statistical Mechanics (Wiley, New York, 1987).

[19] T. L. Ho and E. J. Mueller, Phys. Rev. Lett. 92, 160404 (2004).

[20] R. P. Feynman, Phys. Rev. 91, 1291 (1953). 